\title{
Possibilities of enhancing the neuroprotective effect of autophagy activation in the brain by stimulation of an mTOR-independent pathway of its regulation in a transgenic mouse model of Parkinson's disease
}

Anna A. Akopyan

Scientific Research Institute of

Physiology and Basic Medicine

Novosibirsk, Russia

annaaleksanovna@mail.ru
Aleksandr B. Pupyshev

Scientific Research Institute of

Physiology and Basic Medicine

Novosibirsk, Russia

apupyshev@mail.ru
Maria A. Tikhonova

Scientific Research Institute of Physiology and Basic Medicine

Novosibirsk, Russia

tikhonovama@physiol.ru
Abstract - Autophagy induction promotes cell survival that is especially important for neurons which have a limited proliferative resource. Autophagy is regulated by the classical mTOR-dependent mechanism activated by rapamycin, and also via mTOR-independent pathways triggered by trehalose, lithium, metformin, etc. The neuroprotective effect was shown upon combined activation of these pathways in vitro. However, the possibilities of enhancing the therapeutic effect of autophagy activation in vivo remain unclear.

Transgenic mice of B6.Cg-Tg (Prnp$\mathrm{SNCA}^{*} \mathrm{A53T)23Mkle/J}$ strain with overexpression of alphasynuclein were used as a model of Parkinson's disease (PD) in the study. In the striatum and substantia nigra, which are mainly affected in PD, trehalose caused an increase in autophagy, as measured by the expression of the autophagy marker LC3-II. Trehalose in combination with rapamycin increased LC3-II expression by two to three times in comparison with the action of rapamycin alone. In the frontal cortex no changes in LC3-II expression were observed neither under the action of trehalose nor with the combined treatment with trehalose and rapamycin. The most pronounced neuroprotective effect was observed upon the combined use of rapamycin and trehalose by the tyrosine hydroxylase expression in the nigrostriatal system marking the restoration of dopaminergic neurons. In the Rotarod test, the mice were tested for motor function. Animals treated with rapamycin, trehalose, or their combination stayed much longer on a rotating drum, compared to controls. Autophagy contribution to the therapeutic effects was confirmed by administration of autophagy inhibitor 3-methyladenine, which completely blocks the neuroprotective effects of the drugs.

Key words - autophagy, rapamycin, trehalose, mice, alpha synuclein, Parkinson's disease

\section{ACKNOWLEDGMENT}

This work was supported by a grant No. 16-04-01423 from the Russian Foundation for Basic Research. 